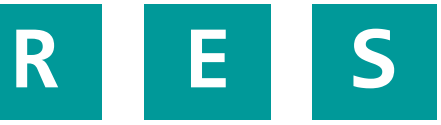 \\ $P$ \\ Y \\ $\mathrm{N}$

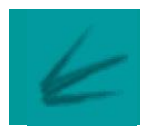 \\ Prespon
}

Revista Salud Pública y Nutrición

\section{FUNCIONALIDAD FAMILIAR Y DEPRESIÓN EN ADULTOS EN LA ATENCIÓN PRIMARIA}

FAMILY FUNCTIONALITY AND DEPRESSION IN ADULTS IN PRIMARY CARE

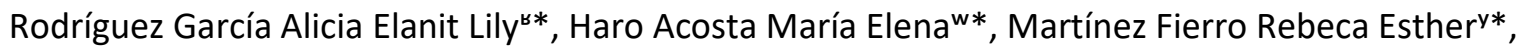
Ayala Figueroa Rafael Iván*, Román Matus Alexis ${ }^{\text {h* }}$

${ }^{\text {} U n i d a d ~ d e ~ M e d i c i n a ~ F a m i l i a r ~ N o . ~} 9$ del Instituto Mexicano del Seguro Social. " Delegación Baja California del Instituto Mexicano del Seguro Social. ' Unidad de Medicina Familiar No. 40 del Instituto Mexicano del Seguro Social. Universidad Autónoma de Baja California. Facultad de Medicina, Mexicali. ' hospital General de Zona No. 30 del Instituto Mexicano del Seguro Social.

*Mexicali, Baja California, México.

Citation: Rodríguez García AEL., Haro Acosta ME., Martínez Fierro RE., Ayala Figueroa RI., Román Matus A. (2017) Funcionalidad familiar y depresión en adultos en la atención primaria. Revista de Salud Pública y Nutrición, 16(4), 20-23.

Editor: Esteban G. Ramos Peña, Dr. CS., Universidad Autónoma de Nuevo León, Facultad de Salud Pública, Monterrey Nuevo León, México.

Copyright: (C2017 Rodríguez García AEL. et al. This is an open-access article distributed under the terms of Creative Commons Attribution License [CC BYND 4.0], which permits unrestricted use, distribution, and reproduction in any medium, provided the original author and source are credited.

Competing interests: The authors have declared that no competing interests exist.

DOI: https://doi.org/10.29105/respyn16.4-3

Email: Lily rodriguitos@hotmail.com 


\title{
FUNCIONALIDAD FAMILIAR Y DEPRESIÓN EN ADULTOS EN LA ATENCIÓN PRIMARIA
}

\author{
Rodríguez García Alicia Elanit Lily*, Haro Acosta María Elena ${ }^{\mathrm{w} *}$, Martínez Fierro Rebeca Esther*, Ayala \\ Figueroa Rafael Iván`*, Román Matus Alexis ${ }^{\text {h* }}$
}

\begin{abstract}
${ }^{\text {घUnidad de Medicina Familiar No. } 9 \text { del Instituto Mexicano del Seguro Social. " } D e l e g a c i o ́ n ~ B a j a ~ C a l i f o r n i a ~ d e l ~ I n s t i t u t o ~}$ Mexicano del Seguro Social. YUnidad de Medicina Familiar No. 40 del Instituto Mexicano del Seguro Social. ^Universidad Autónoma de Baja California. Facultad de Medicina, Mexicali. h Hospital General de Zona No. 30 del Instituto Mexicano del Seguro Social.
\end{abstract}

*Mexicali, Baja California, México.

\section{RESUMEN}

Introducción: La depresión es un trastorno emocional con estado de abatimiento e infelicidad, predominan síntomas afectivos. La Funcionalidad Familiar es la dinámica interactiva y sistemática que se da entre los miembros de una familia. Objetivo: Determinar la funcionalidad familiar y depresión en adultos en la atención primaria. Material y Métodos: Estudio transversal en 176 adultos de 20 a 60 años de edad seleccionados a conveniencia, en el período noviembre 2015 a mayo 2016. Las variables evaluadas fueron sexo, estado civil, edad, funcionalidad familiar con el Test de Percepción del Funcionamiento Familiar y el grado de depresión con el Test Mayor Depresión. Se utilizó estadística descriptiva y prueba de chi cuadrada con el programa Statistical Package for the Social Sciences 21. Resultados: Las Familias Funcionales fueron el 38.6\% y las Familias Moderadamente Funcionales el $23.3 \%$ sin depresión. La depresión severa predominó en las Familias Moderadamente Funcionales en el 7.3\%, seguidas de las Familias Disfuncionales 3.9\% y en las Severamente disfuncionales 3.9\%. Se observó relación estadísticamente significativa entre la funcionalidad familiar y la depresión $(p=0.001)$. Conclusiones: La funcionalidad familiar está relacionada con el grado de depresión; influye en presencia o ausencia de depresión en los adultos en etapa productiva.

Palabras clave: familia, depresión, adulto

\section{ABSTRACT}

Introduction: Depression is an emotional disorder that presents itself as a state of dejection and unhappiness where affective symptoms predominate. Family Functionality is the interactive and systematic dynamics that occurs among the members of a family. Objective: To determine the family functionality and adult depression in primary. Methods: Transversal study, in 176 adults between the ages of 20 and 60 years, selected by convenience in the period from November 2015 to May 2016 . The variables evaluated were gender, marital status, age, family functionality with the Perception of Family Functioning Test and the degree of depression with the Major Depression Test. They were analyzed with descriptive and inferential statistics with chi-square test with the Statistical Package for the Social Sciences 21. Results: Functional families were $38.6 \%$ and Moderately Functional Families were 23.3\% without depression. Severe depression predominated Moderately Functional Families $7.3 \%$ followed by the Dysfunctional Families 3.9\% and in the Severely Dysfunctional $3.9 \%$. There was a statistically significant relationship between family function and depression $(p=0.001)$. Conclusions: Family functionality is related with the degree of depression; influences the presence or absence of depression in productive adults.

Key words: family, depression, adult. 


\section{Introducción}

La depresión es un trastorno emocional que se presenta como un estado de abatimiento e infelicidad donde predominan síntomas afectivos, de tipo cognitivo, volitivo o incluso somático (Vihang 2013, Black 2015). La Funcionalidad Familiar es la dinámica interactiva y sistemática que se da entre los miembros de una familia y se evalúan a través de las categorías de cohesión, armonía, permeabilidad, afectividad, participación y adaptabilidad (Ortiz 2005, Castellón 2012).

En un trabajo realizado por Nam (2016) en 304 refugiados de Corea del Norte en el Sur de Corea se encontró que, el $44 \%$ presentaban depresión y demostraron que al mejorar los vínculos familiares hay reducción de la depresión. Recientemente, Lu (2017) valoró el efecto de la depresión con la funcionalidad familiar y resiliencia en 474 adultos mayores en China, observó una correlación negativa entre la depresión con la funcionalidad familiar y la resiliencia.

El estudio transversal realizado en dos secundarias privadas y públicas en Venezuela en 1,082 adolescentes de 14 a 19 años de edad para establecer la relación entre depresión y funcionalidad familiar, mostró que el $40 \%$ posee una alta funcionalidad familiar y el $30 \%$ no registró depresión. El estudio concluye que los adolescentes no poseen depresión, tienen alta funcionalidad familiar y no existen diferencias estadísticamente significativas al relacionar los niveles de depresión con la funcionalidad familiar (Salinas 2013). En México se determinó la frecuencia de depresión y disfunción familiar en 252 adolescentes en una unidad de medicina familiar, se observó una frecuencia de depresión en las mujeres fue de $42.4 \%$ y en los hombres de $18.7 \%$ (Leyva 2007).

Existe poca evidencia publicada de la presencia de depresión y funcionalidad familiar en adultos de 20 a 60 años, por lo que el objetivo es conocer la funcionalidad familiar en pacientes adultos con depresión en la Unidad de Medicina Familiar No. 09 del Instituto Mexicano Seguro Social en Mexicali Baja California.

\section{Material y Métodos}

Estudio transversal en 176 adultos de 20 a 60 años, en ambos de géneros, que acudieron a la consulta externa de la Unidad de Medicina Familiar No. 09, del Instituto Mexicano del Seguro Social en Mexicali Baja California, en el período de noviembre 2015 a mayo 2016.

La muestra se calculó de acuerdo a la fórmula de proporciones, los pacientes fueron seleccionados conforme acudieron a consulta, a los participantes se les solicitó firmar el consentimiento informado. El estudio fue aprobado por el Comité de Investigación. Los criterios de exclusión comprendieron pacientes con problemas mentales y usuarios de drogas. A la población de estudio se le aplicó el Test de Depresión Mayor MDI (Olsen 2003, Bech 2015) y Percepción del Funcionamiento Familiar FF-SIL. El puntaje del Test de Depresión Mayor MDI es: sin depresión menos de 20, depresión leve 20 a 24 puntos, moderada 25 a 29 y severa 30 o más, el puntaje del FF-SIL es: Familia Funcional 70-57 puntos, Familia Moderadamente Funcional 56-43, Familia Disfuncional 42-28, Familia Severamente Disfuncional 27-14 (Pérez 1997, Fuentes 2016).

Se realizó un análisis descriptivo e inferencial (chicuadrada para variables cualitativas), se consideró significativa una $\mathrm{p}<0.05$. Los datos se analizaron en el programa SPSS v. 21.

\section{Resultados}

De los 176 adultos de 20 a 60 años de edad que acudieron a consulta externa de la Unidad de Medicina Familiar No.9; el 75\% ( $n=132)$ fueron mujeres y el $25 \%(n=44)$ hombres. La media de edad de las mujeres fue $39.23 \pm 10.45$ (1DS) y de los hombres de $36.98 \pm 11.32$ (1DS), el $28.4 \%$ tuvo en 20 y 30 años, el $26.7 \%$ entre 31 y 40 años, $29.5 \%$ entre 41 y 50 años (Tabla No. 1).

\begin{tabular}{|c|c|c|c|c|c|c|c|c|c|c|}
\hline \multirow[b]{2}{*}{ Género } & \multicolumn{2}{|c|}{20 a 30} & \multicolumn{2}{|c|}{31 a 40} & \multicolumn{2}{|c|}{41 a 50} & \multicolumn{2}{|c|}{51 a 60} & \multicolumn{2}{|c|}{ Total } \\
\hline & No. & $\%$ & No. & $\%$ & No. & $\%$ & No. & $\%$ & No. & $\%$ \\
\hline Femenino & 33 & 64.7 & 38 & 80.9 & 41 & 78.8 & 20 & 76.9 & 132 & 75 \\
\hline Masculino & 18 & 35.3 & 9 & 19.1 & 11 & 21.2 & 6 & 23.1 & 44 & 25 \\
\hline Total & 51 & 100.0 & 47 & 100.0 & 52 & 100.0 & 26 & 100.0 & 176 & 100 \\
\hline
\end{tabular}

De acuerdo al estado civil el $60.8 \%$ eran casados ( $21.5 \%$ hombres y $78.5 \%$ mujeres), $17 \%$ solteros ( $36.7 \%$ hombres y $63.3 \%$ mujeres), $13.6 \%$ en unión libre. (Tabla No. 2). 
Tabla 2. Población por género según estado civil

\begin{tabular}{lcccccc}
\hline Edo. Civil & \multicolumn{2}{c}{ Hombres } & \multicolumn{2}{c}{ Mujeres } & \multicolumn{2}{c}{ Total } \\
& No. & $\%$ & No. & $\%$ & No. & $\%$ \\
\hline Soltero & 11 & 25.0 & 19 & 14.4 & 30 & 17.0 \\
Casado & 23 & 52.3 & 84 & 63.6 & 107 & 60.8 \\
Unión Libre & 9 & 20.5 & 15 & 11.4 & 24 & 13.6 \\
Separado & 1 & 2.3 & 4 & 3.0 & 5 & 2.8 \\
Viudo & 0 & 0.0 & 9 & 6.8 & 9 & 5.1 \\
Divorciado & 0 & 0.0 & 1 & 0.8 & 1 & 0.6 \\
Total & 44 & 100 & 132 & 100.0 & 176 & 100.0 \\
\hline Fuente: Encuesta & & & & & &
\end{tabular}

El $43.2 \%$ de la población de estudio, refiere tener familias funcionales $y$ el $37.5 \%$ familias moderadamente funcionales. En cuanto a la depresión: el $71.0 \%$ de la población de estudio no la presentó. De acuerdo a la funcionalidad familiar y depresión: el $38.6 \%$ de las familias funcionales y el 23.3\% moderadamente funcionales no presentaron depresión. La depresión severa se observó en el 7.4\% en familias moderadamente funcional; disfuncionales y severamente disfuncionales en el $4.0 \%$. Al relacionar la funcionalidad familiar con el grado de depresión con la prueba de chi cuadrada fue estadísticamente significativa con $\mathrm{p}=0.001$ (Tabla No. 3).

Tabla 3. Población por grado de depresión y funcionalidad familiar

\begin{tabular}{|c|c|c|c|c|c|c|c|c|c|c|}
\hline \multirow{3}{*}{$\begin{array}{l}\text { Funcionalidad } \\
\text { Familiar }\end{array}$} & \multicolumn{10}{|c|}{ Grado de Depresiòn } \\
\hline & \multicolumn{2}{|c|}{ Sin Depresiòn } & \multicolumn{2}{|c|}{$\begin{array}{l}\text { Depresiòn } \\
\text { leve }\end{array}$} & \multicolumn{2}{|c|}{$\begin{array}{l}\text { Depresiòn } \\
\text { moderada }\end{array}$} & \multicolumn{2}{|c|}{$\begin{array}{c}\text { Depresiòn } \\
\text { severa }\end{array}$} & \multicolumn{2}{|c|}{ Total } \\
\hline & No. & $\%$ & No. & $\%$ & No. & $\%$ & No. & $\%$ & No. & $\%$ \\
\hline Funcional & 68 & 38.6 & 2 & 1.1 & 3 & 1.7 & 3 & 1.7 & 76 & 43.2 \\
\hline Moderado & 41 & 23.3 & 7 & 4.0 & 5 & 2.8 & 13 & 7.4 & 66 & 37.5 \\
\hline Disfuncional & 14 & 8.0 & 1 & 0.6 & 2 & 1.1 & 7 & 4.0 & 24 & 13.6 \\
\hline Severa & 2 & 1.1 & 1 & 0.6 & 0 & 0.0 & 7 & 4.0 & 10 & 5.7 \\
\hline Total & 125 & 71.0 & 11 & 6.3 & 10 & 5.7 & 30 & 17.0 & 176 & 100.0 \\
\hline
\end{tabular}

Referente a los grados de funcionalidad de las familias, el estado civil de casado, es el que presenta el mayor porcentaje en todos los grados mencionados, en especial en familias funcionales y moderadamente funcional (Tabla No. 4).

\begin{tabular}{cccccccccccc}
\multicolumn{10}{c}{ Tabla 4. Población por Funcionalidad Familiar según estado Civil } \\
\hline Edo.Civil & $\begin{array}{c}\text { Familiar } \\
\text { Funcional }\end{array}$ & $\begin{array}{c}\text { Moderadamente } \\
\text { Funcional }\end{array}$ & $\begin{array}{c}\text { Disfuncional } \\
\text { Severamente } \\
\text { Disfuncional }\end{array}$ & \multicolumn{2}{c}{ Total } \\
& No. & $\%$ & No. & $\%$ & No. & $\%$ & No. & $\%$ & No. & $\%$ \\
\hline Soltero & 9 & 11.8 & 14 & 21.2 & 5 & 20.8 & 2 & 20.0 & 30 & 17.0 \\
Casado & 50 & 65.8 & 42 & 63.6 & 9 & 37.5 & 6 & 60.0 & 107 & 60.8 \\
Unión libre & 10 & 13.2 & 8 & 12.1 & 5 & 20.8 & 1 & 10.0 & 24 & 13.6 \\
Separado & 2 & 2.6 & 1 & 1.5 & 2 & 8.3 & 0 & 0.0 & 5 & 2.8 \\
Viudo & 5 & 6.6 & 0 & 0.0 & 3 & 12.5 & 1 & 10.0 & 9 & 5.1 \\
Divorciado & 0 & 0.0 & 1 & 1.5 & 0 & 0.0 & 0 & 0.0 & 1 & 0.6 \\
& 76 & 100.0 & 66 & 100.0 & 24 & 100.0 & 10 & 100.0 & 176 & 100.0 \\
\hline Fuente: Encuesta & & & & & & & & & & &
\end{tabular}

A excepción del grado moderado de depresión, el estado civil de casado tiene el mayor porcentaje en todos los grados de depresión (Tabla No.5).

\begin{tabular}{lcccccccccccc}
\multicolumn{10}{c}{ Tabla 5. Población por grado de depresión y estado civil } \\
\hline Edo.Civil & \multicolumn{1}{c}{ Sin Depresión } & $\begin{array}{c}\text { Depresión } \\
\text { Leve }\end{array}$ & \multicolumn{3}{c}{$\begin{array}{c}\text { Depresión } \\
\text { Moderada }\end{array}$} & $\begin{array}{c}\text { Depresión } \\
\text { Severa }\end{array}$ & \multicolumn{2}{c}{ Total } \\
& No. & $\%$ & No. & $\%$ & No. & $\%$ & No. & $\%$ & No. & $\%$ \\
\hline Soltero & 19 & 10.8 & 0 & 0.0 & 5 & 2.8 & 6 & 3.4 & 30 & 17.0 \\
Casado & 80 & 45.5 & 8 & 4.5 & 2 & 1.1 & 17 & 9.7 & 107 & 60.8 \\
Unión libre & 16 & 9.1 & 3 & 1.7 & 2 & 1.1 & 3 & 1.7 & 24 & 13.6 \\
Separado & 4 & 2.3 & 0 & 0.0 & 0 & 0.0 & 1 & 0.6 & 5 & 2.8 \\
Viudo & 6 & 3.4 & 0 & 0.0 & 0 & 0.0 & 3 & 1.7 & 9 & 5.1 \\
Divorciado & 0 & 0.0 & 0 & 0.0 & 1 & 0.6 & 0 & 0.0 & 1 & 0.6 \\
Total & 125 & 71.0 & 11 & 6.3 & 10 & 5.7 & 30 & 17.0 & 176 & 100 \\
\hline Fuente: Encuesta & & & & & & & & & &
\end{tabular}

\section{Discusión}

En el presente estudio se observa baja frecuencia de la alteración de la funcionalidad familiar en los pacientes con depresión en la unidad de medicina familiar. Existe poca evidencia de estudios en adultos de 20 a 60 años.

Predominaron las familias funcionales, comparándolo con un estudio realizado en Estado Unidos en adultos mayores de 60 años observaron síntomas depresivos disminuidos en las familias funcionales (Keeler 2016).

Otro estudio en adultos mayores realizado en Tamaulipas mostró que la Funcionalidad Familiar no está relacionada con el grado de depresión, pero se documentó que la familia nuclear es un factor protector para la disfuncionalidad familiar (Saavedra 2016).

\section{Conclusiones}

En las Familias Funcionales existe menor frecuencia de depresión comparadas con las Moderadamente Funcional, en estas últimas se observó la presencia de depresión severa en la población de adultos de 20 a 60 años.

\section{Agradecimientos}

Al Instituto Mexicano del Seguro Social, por otorgar el permiso y a los pacientes que quisieron participar en el estudio.

\section{Bibliografía}

Bech P, Timmerby N, Martiny K, Lunde M, Soendergaard S. 2015.Psychometric evaluation of the Major Depression Inventory (MDI) as depression severity scale using the 
LEAD (Longitudinal Expert Assessment of All Data) as index of validity. BMC Psychiatry; 15:190.

Black DW. 2015, Manual diagnóstico y estadístico de los trastornos mentales DSM-V. 5ta Ed. Barcelona España, Masson,

Castellón S, Ledesma A. Julio 2012 El funcionamiento familiar y su relación con la socialización infantil. CCCSS. [Consultado el 14 de mayo de 2017]. Disponible en: www.eumed.net/rev/cccss/21/

Fuentes Aguilar AP, Merino Escobar JM. 2016. Validación de un instrumento de funcionalidad familiar [tesis]. Universidad de Concepción.

Keeler AN, Siegel J. 2016. Depression, help-seeking perceptions, and perceived family functioning among Spanish-Dominant Hispanics and NonHispanic Whites. Journal of Affective Disorders ; 202:236-246.

Leyva Jiménez R, Hernández Juárez AM, Nava Jiménez G, López Gaona V.2007. Depresión en adolescentes y funcionamiento familiar. Revista Médica del Instituto Mexicano del Seguro Social; 45(3):225-232.

Lu C, Yuan L, Lin W, Zou Y, Pan S.2017. Depression and resilience mediates the effect of family function on quality of life of the elderly. Archives of Gerontology and Geriatrics. 71:34-42.

Nama B, Kim J, De Vylder JE, Song A. 2016. Family functioning, resilience, and depression among North Korean refugees. Psychiatry Res.245:451-7.

Olsen LR, Jensen DV, Noerholm V, Martiny K, Bech P.2003 The internal and external validity of the Major Depression Inventory in measuring severity of depressive states Psychol Med; 33(2):351-356.

Ortiz Gómez MT, Padilla Torres MA, Ortiz Padilla E.2005. Diseño de intervención en funcionamiento familiar. Psicol Am Lat. No.3. [consultado el 14 de abril de 2017].

Disponible en:

pepsic.bvsalud.org/scielo.php?script=sci_artte $\mathrm{xt} \&$ pid=S1870-350X

Pérez E, De la Cuesta D, Louro I, Bayarre H1997 Funcionamiento familiar Construcción y validación de un instrumento. Ciencias de la Salud Humana; 4(1)63-66.

Saavedra González AG, Rangel Torres SL, García de León A, Duarte Ortuño A, Bello Hernández YE, Infante Sandoval A.2016. Depresión y funcionalidad familiar en adultos mayores de una unidad de medicina familiar de Guayalejo, Tamaulipas, México. Atención Familiar. (23):24-28.

Salinas A, Alonso S, Quintero O, Hernández M, Molina L, Lozada R et al.2013. Relación entre depresión y funcionalidad familiar en adolescentes de instituciones educativas públicas y privadas. Academia. 12(28):31724.

Vihang N. 2013. American Psychiatric Association. Diagnostic and Statistical Manual of Mental Disorders. 5th ed. Arlington, VA: American Psychiatric Publishing. 